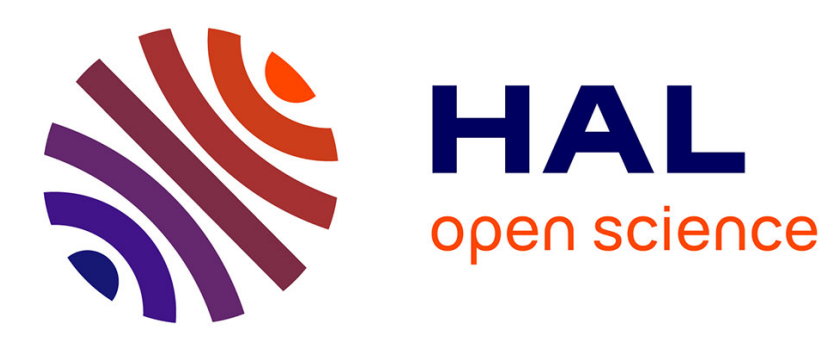

\title{
Les mutations de l'industrie aéronautique civile française: concentration, externalisation et firme-pivot
} Med Kechidi, Damien Talbot

\section{To cite this version:}

Med Kechidi, Damien Talbot. Les mutations de l'industrie aéronautique civile française: concentration, externalisation et firme-pivot. Entreprises et Histoire, 2014, Brève histoire, 2013/4 (73), pp.75-88. hal-01713143

\section{HAL Id: hal-01713143 \\ https://hal.science/hal-01713143}

Submitted on 20 Feb 2018

HAL is a multi-disciplinary open access archive for the deposit and dissemination of scientific research documents, whether they are published or not. The documents may come from teaching and research institutions in France or abroad, or from public or private research centers.
L'archive ouverte pluridisciplinaire HAL, est destinée au dépôt et à la diffusion de documents scientifiques de niveau recherche, publiés ou non, émanant des établissements d'enseignement et de recherche français ou étrangers, des laboratoires publics ou privés. 


\title{
REVUE ENTREPRISE ET HISTOIRE
}

$2013 / 4\left(n^{\circ} 73\right)$

\section{LES MUTATIONS DE L'INDUSTRIE AERONAUTIQUE CIVILE FRANÇAISE : CONCENTRATION, EXTERNALISATION ET FIRME-PIVOT}

\author{
Med KECHIDI \\ Professeur en Sciences de Gestion, HDR, Framespa, UT2J, \\ med.kechidi@univ-tlse2.fr
}

Damien TALBOT

Professeur en Sciences de Gestion, CRCGM, Ecole Universitaire de Management, Université d'Auvergne.

\section{Introduction}

Historiquement dominée par le fait militaire, l'aéronautique est une industrie où l'intervention publique est de règle. Même si les évolutions récentes marquent un net fléchissement des liens avec les politiques d'arsenal, les considérations de défense ont de tout temps motivées l'implication des Etats vis-à-vis de cette industrie. En France, l'histoire récente de cette industrie est étroitement liée à celle de l'aéronautique européenne. Les grands industriels aéronautiques actuels se sont constitués sur la base d'un long mouvement de concentration fait de fusions et d'acquisitions, d'abord dans un cadre national puis européen, coopérations menées ou encouragées par les Etats. D’un point de vue organisationnel, nous sommes passés d'un ensemble très éclaté d'entreprises nationales à une consolidation européenne aboutissant à la création d'un acteur unique, EADS.

Plus précisément, dès les années 1930, l'Etat français impose la rationalisation de l'industrie. $\mathrm{Au}$ sortir de la Première Guerre mondiale, la répartition des charges de travail entre de nombreuses entreprises exigée par les militaires afin d'éviter toute constitution de monopoles, conduit à morceler l'industrie aéronautique française. Cette dispersion des efforts se traduit par d'importants problèmes de qualité qui affectent des appareils généralement peu fiables, sans parler des surcoûts induits par les duplications industrielles. A partir de 1927, la situation économique ainsi que la perspective d'un nouveau conflit mondial provoquent un 
infléchissement de cette politique publique de soutien. La rationalisation passe avant tout par la diminution du nombre des usines : il y a encore en 1930 vingt-cinq firmes créatrices d'avions, et autant de bureaux d'études (Chadeau 1987¹). L'Etat décide donc que les commandes significatives n'iront qu'à des sociétés regroupées.

L'arrivée au pouvoir du Front Populaire en 1936 marque un tournant pour cette industrie, l'Etat nationalisant les deux tiers des avionneurs tout en leur apportant une aide financière. Ces nationalisations apparaissent comme un moyen d'imposer des regroupements, desquels doit naître une industrie aéronautique capable de produire rapidement un grand nombre d'avions performants. La guerre interrompt alors un mouvement qui reprendra dans les années 1950. La répartition des programmes pratiquée à outrance n'est plus tenable dans un contexte d'ouverture des frontières, les entreprises françaises étant maintenues dans une situation d'infériorité face à la concurrence américaine. Il s'avère indispensable de regrouper les firmes nationales autour des programmes pour constituer des champions nationaux.

Cette logique de concentration de l'industrie s'observe également au niveau européen. En juillet 2000 la naissance de l'entreprise European Aeronautic Defence and Space Company (EADS) vient parachever les restructurations nationales. Elle est constituée de la fusion des activités aéronautiques, espace et défense de trois groupes européens : 1'Allemand Daimler Aerospace AG, le Français Aerospatiale Matra et l'Espagnol CASA. Cette fusion marquait, par la première fois dans l'histoire de cette industrie, la naissance d'une entreprise aéronautique et spatiale multinationale. Aujourd'hui, Airbus SAS, filiale phare du groupe EADS, regroupe quatre entités nationales: Airbus France, Airbus Deutschland, Airbus Espana, Airbus UK. Ces sociétés employaient, à la fin de l'année 2012, plus de 54000 personnes et disposent d'actifs industriels répartis sur 16 sites européens.

L'ensemble du secteur est aujourd'hui organisé autour de quelques grands groupes industriels : EADS-Airbus pour les avions civils et Dassault Aviation pour les avions militaires, Eurocopter (filiale de EADS) pour les hélicoptères civils et militaires, Safran dont les filiales SNECMA et Turbomeca dominent le secteur de la motorisation, Arianespace leader mondial du lancement des satellites, Thales Alenia Space acteur mondial dans le domaine de l'infrastructure orbitale.

A ces leaders mondiaux, il faut ajouter des firmes de moindre dimension mais qui constituent des maillons essentiels dans les processus de production d'avions, de satellites ou de missiles. On peut citer Latécoère (aérostructures et câblage électrique), Goodrich Aerospace (nacelles

\footnotetext{
${ }^{1}$ E. Chadeau, De Blériot à Dassault : L'Industrie aéronautique en France, 1900-1950, Paris, Fayard, 1987.
} 
et systèmes embarqués notamment), Labinal (filiale Safran pour le câblage électrique), les filiales de Safran Messier Buggati et Messier-Dowty (trains d'atterrissage et moteurs), Thales Avionics et Liebherr Aerospace pour les systèmes embarqués. On le devine, cette industrie a connu de nombreux bouleversements qui ont remodelés son organisation en profondeur.

Les développements qui suivent décrivent ces transformations organisationnelles à l'œuvre dans cette industrie et les logiques qui les sous-tendent. Nous montrons d'abord comment s'est déroulé le passage d'une logique d'arsenal, caractéristique des programmes des années 1950 et 1960, à une logique de marché inaugurée par les premiers succès commerciaux d'Airbus. Ensuite, nous mettons en avant la trajectoire d'innovation suivie par les programmes Airbus, illustrant une certaine histoire industrielle de l'avionneur européen. Dans un troisième temps, nous caractérisons le nouveau modèle d'organisation industrielle qui s'est progressivement mis en place.

\section{Des années 1950 à 2000 : des champions nationaux à un leader mondial}

Deux époques structurent l'histoire de l'industrie aéronautique civile. La première, dite de «logique d'arsenal » (Muller, 1988²) va du début du XX ${ }^{\mathrm{e}}$ siècle jusqu'aux années 1970 et renvoie à une industrie dans laquelle l'Etat est l'acteur clé. La seconde débute en Europe avec le programme Airbus. Elle est dite de « logique de marché » et voit le rôle de l'Etat peu à peu se réduire au profit des avionneurs européens qui définissent les programmes en fonction des attentes des compagnies aériennes, l'Etat poursuivant son soutien financier. La création d'EADS en 2000 finalise cette évolution entamée avec le GIE Airbus.

\subsection{Une industrie sous tutelle étatique : la logique d'arsenal}

La logique d'arsenal qualifie une industrie de souveraineté où prédominent les enjeux technologiques. Concrètement, un avion efficient est un avion performant d'un point de vue d'abord technique, reléguant au second plan les nécessités commerciales. Dans cette logique, l'Etat demeure l'acteur central de l'industrie pour plusieurs raisons. Premièrement, l'aéronautique est un secteur dit de «frontière technologique » dans lequel tout pays industrialisé se doit de figurer (Muller, $1988^{3}$ ). Deuxièmement, ce secteur provoquerait des retombées bénéfiques à l'ensemble de l'industrie nationale. Par conséquent, les ressources publiques mobilisées par cette industrie augmenteraient la compétitivité du pays (Moura,

\footnotetext{
${ }^{2}$ P. Muller, Airbus l'ambition européenne, logique d'Etat, logique de marché, Paris, L'Harmattan, 1988.

${ }^{3}$ Ibid.
} 
$2007^{4}$ ). Troisièmement, le caractère militaire d'une partie de cette industrie conduit l'ensemble de cette dernière à être placé sous tutelle étatique, puisque selon la représentation dominante en vigueur «il faut que la défense de la France soit française $»^{5}$.

Par conséquent, l'Etat cherche à maîtriser ce secteur considéré comme vital pour la nation, tandis que les industriels sont dans l'attente de décisions gouvernementales pour agir. Depuis le début du $\mathrm{XX}^{\mathrm{e}}$ siècle en effet, l'Etat désigne le ou les constructeurs et indique les caractéristiques de l'avion à construire. Il est client et actionnaire des entreprises qui réalisent les programmes, dont il est le premier financeur. Enfin, il organise la consolidation de l'industrie nationale en créant des champions nationaux : création de la SNIAS (nommée Aerospatiale par la suite), de la SNECMA (aujourd'hui intégrée à Safran) et consolidation de Dassault ( $c f$. figure 1).

Figure 1. Les champions nationaux français

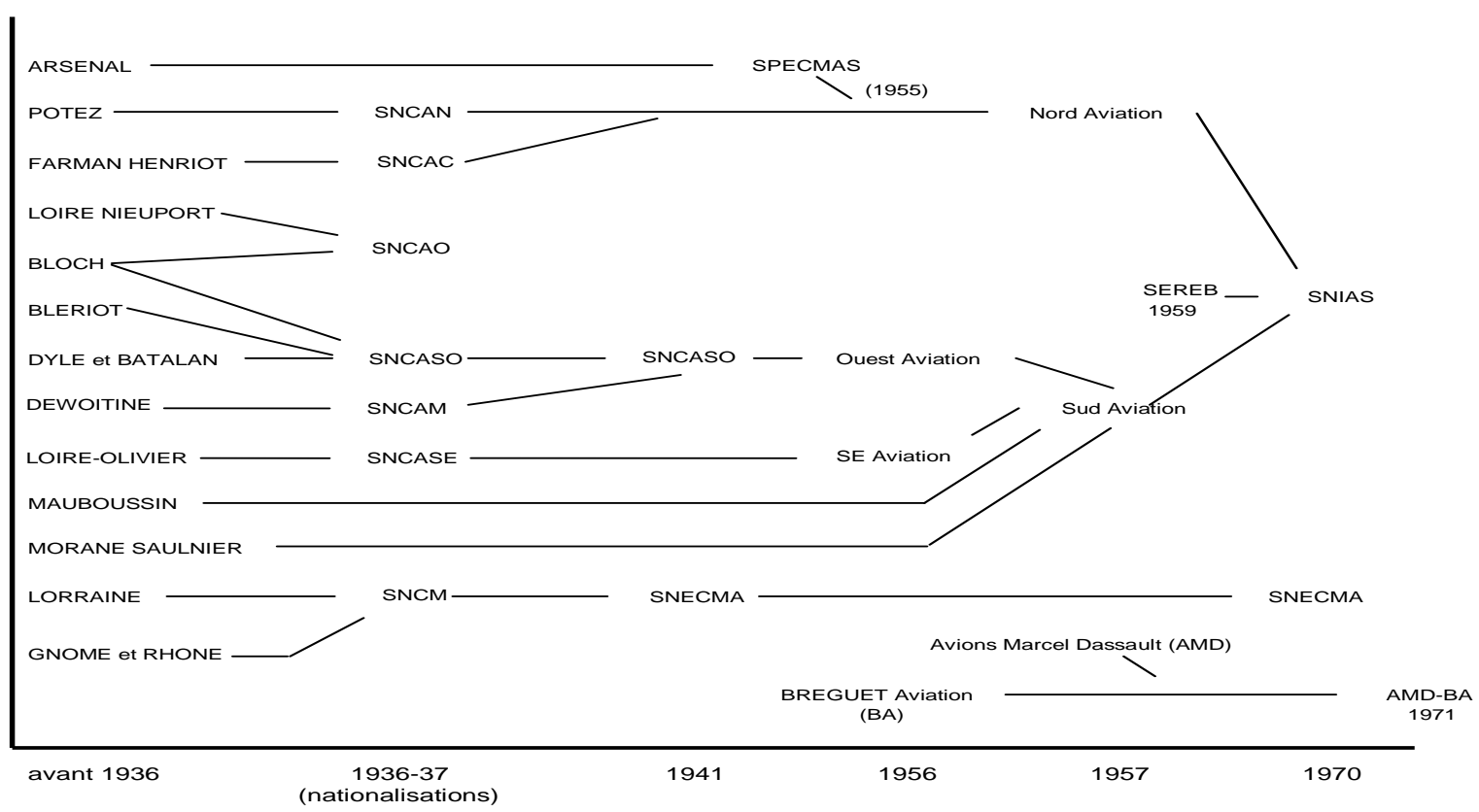

Source : à partir d'Y. Morvan (1983) ${ }^{6}$.

Dans un contexte de préservation de l'indépendance nationale, les coopérations européennes doivent se réaliser sur une répartition égalitaire des rôles et par programme. Dans le cas le plus courant d'une coopération bipartite, cela signifie que les coûts, les charges de travail

\footnotetext{
${ }^{4} \mathrm{~S}$. Moura, L'impossible banalisation des industries de défense. L'apport d'une analyse institutionnaliste à l'étude de la dynamique des industries, thèse de doctorat en Sciences Economiques, Université Montesquieu Bordeaux IV, septembre, 2007.

${ }^{5}$ Selon les termes gaulliens, repris p. 150 par Moura, ibid.

${ }^{6}$ Y Morvan, "L'aéronautique : une industrie performante face à des contraintes accrues", dans "L'industrie en France", sous la direction de B. Bellon Et J-M. Chevalier, Ed. Flammarion, 1983, p. 255.
} 
induites ainsi que les bénéfices éventuels sont divisés à parts égales (Beckouche, 1996 ${ }^{7}$ ). Les raisons de la mise en place de telles coopérations sont multiples : mise en commun de savoirfaire, de technologies, d'un potentiel industriel, d'un marché domestique, ou encore partage des coûts et des risques, mais aussi participation à la construction de l'Union Européenne (Baccrabere, Jorre, 1966 ; Sparaco, 1996 ${ }^{8}$ ).

\subsection{Emergence de la logique de marché}

A partir des années 1960, en raison des échecs commerciaux plus ou moins sévères que connaissait l'industrie aéronautique, la logique d'arsenal est de moins en moins acceptée par les industriels (Muller, 19889 ). Ils souhaitent s'émanciper d'un Etat qui lui cherche à réduire son rôle dans cette industrie afin de minimiser ses dépenses. Dorénavant, il se cantonne principalement au rôle de financeur, les avionneurs définissant les caractéristiques et le prix des avions suite à des études de marché.

Dans une logique de marché, un programme est perçu comme une réussite si l'appareil se vend. Il faut voir là un basculement des priorités : la technologie ne doit être utilisée que si elle confère au produit un avantage commercial et non plus pour elle-même (Le Masson, Weil, Hatchuel, $2006^{10}$ ). La maîtrise des coûts devient alors une préoccupation centrale partagée par tous (Beteille, $1995^{11}$ ), car elle conditionne le succès commercial.

Le Groupement d'Intérêt Economique (GIE) Airbus, crée en 1970, illustre ces changements. Cette organisation permet d'offrir une interface unique aux compagnies aériennes et de mettre en place une filiale commune qui assure la répartition des charges de travail et la coordination entre les partenaires (le français Aerospatiale, le britannique BAe, l'espagnol CASA et l'allemand Deutsche Airbus) en fonction de leurs compétences (Ville, 1995 ${ }^{12}$ ). La forme du GIE, sans capital et sans profit, permet d'organiser une coopération tout en évitant la fusion (Thietard, Koenig, 1987 ; Lathiere, $1995^{13}$ ), en ne plaçant aucun des partenaires dans une

\footnotetext{
${ }^{7}$ P. Beckouche, La nouvelle géographie de l'industrie aéronautique européenne, Paris, L'Harmattan, 1996.

${ }^{8}$ G. Baccrabère, G. Jorre, Toulouse terre d'envol, Toulouse, Privat, 1966 ; P. Sparaco, L'industrie aérospatiale française, collection "Que sais-je", Paris, Presses Universitaires de France, 1996.

${ }^{9}$ Op. cit.

${ }^{10}$ P. Le Masson, B. Weil, A. Hatchuel, Les processus d'innovation. Conception innovante et croissance des entreprises, Paris, Lavoisier, 2006.

${ }^{11}$ R. Beiteille, "Le développement de l'avion, stimulation et coopération européenne", in E. Chadeau (Ed.), AIRBUS, un succès industriel européen, Paris, Institut d'Histoire de l'Industrie et Éditions Rive Droite, 1995.

${ }^{12}$ G. Ville, "Fonctionnement et élargissement de la coopération : l'action des industriels partenaires", in E. Chadeau (Ed.), AIRBUS, un succès industriel européen, Paris, Institut d'Histoire de l'Industrie et Éditions Rive Droite, 1995.

${ }^{13} \mathrm{R}$ Thietart. A., C. Koeinig, "Programmes aérospatiaux : la stratégie de l'organisation mutuelle", Revue Française de Gestion, n ${ }^{\circ} 62$, mars-avril-mai, 1987, p. 42-53; B. Lathiere, "Réflexions sur la formule du
} 
position dominante susceptible de lui permettre d'exercer un pouvoir stratégique, tout en s'affranchissant de la tutelle étatique.

Début 2001, le GIE devient une société par actions simplifiées (SAS). Sa création est une réponse aux limites du système GIE. Airbus SAS, maintenant dotée de fonds propres, peut engager directement des investissements à partir de différentes sources (Etats, partenaires, marchés financiers). De plus, les partenaires vendaient jusqu'alors au GIE leurs productions sans que ce dernier n'ait accès au coût réel des éléments produits (Kechidi, $1996^{14}$ ). De fait, les partenaires conservaient de façon opportuniste une partie des bénéfices réels de leurs productions. Face à l'accélération du mouvement de concentration de l'industrie américaine, il faut voir dans Airbus SAS une suite logique du processus d'intégration de l'industrie européenne largement entamé avec la création d'EADS en 2000 (Talbot, 2005 ${ }^{15}$ ). EADS concentre dorénavant les industries aéronautiques allemande, espagnole et française au-delà des seuls avions civils, pour concerner les autres activités aéronautiques (avions militaires, hélicoptères, lanceurs spatiaux, satellites, missiles) ( $c f$. figure 2). En toute logique, Airbus est détenue à $100 \%$ par EADS.

Groupement d'Intérêt Économique (GIE)", in E. Chadeau (Ed.), AIRBUS, un succès industriel européen, Paris, Institut d'Histoire de l'Industrie et Éditions Rive Droite, 1995.

${ }^{14}$ M. Kechidi, "Coordination inter-entreprises et relations de sous-traitance: le cas d'AEROSPATIALE", Revue d'Économie Régionale et Urbaine, $\mathrm{n}^{\circ} 1,1996$, p. 99-120.

${ }^{15}$ D. Talbot, "Les proximités, entre contrainte et libération de l'action : le cas d'EADS", Economie et Institutions, $\mathrm{n}^{\circ} 6$ et 7, 1er et 2e semestres, 2005, p. 153-180. 


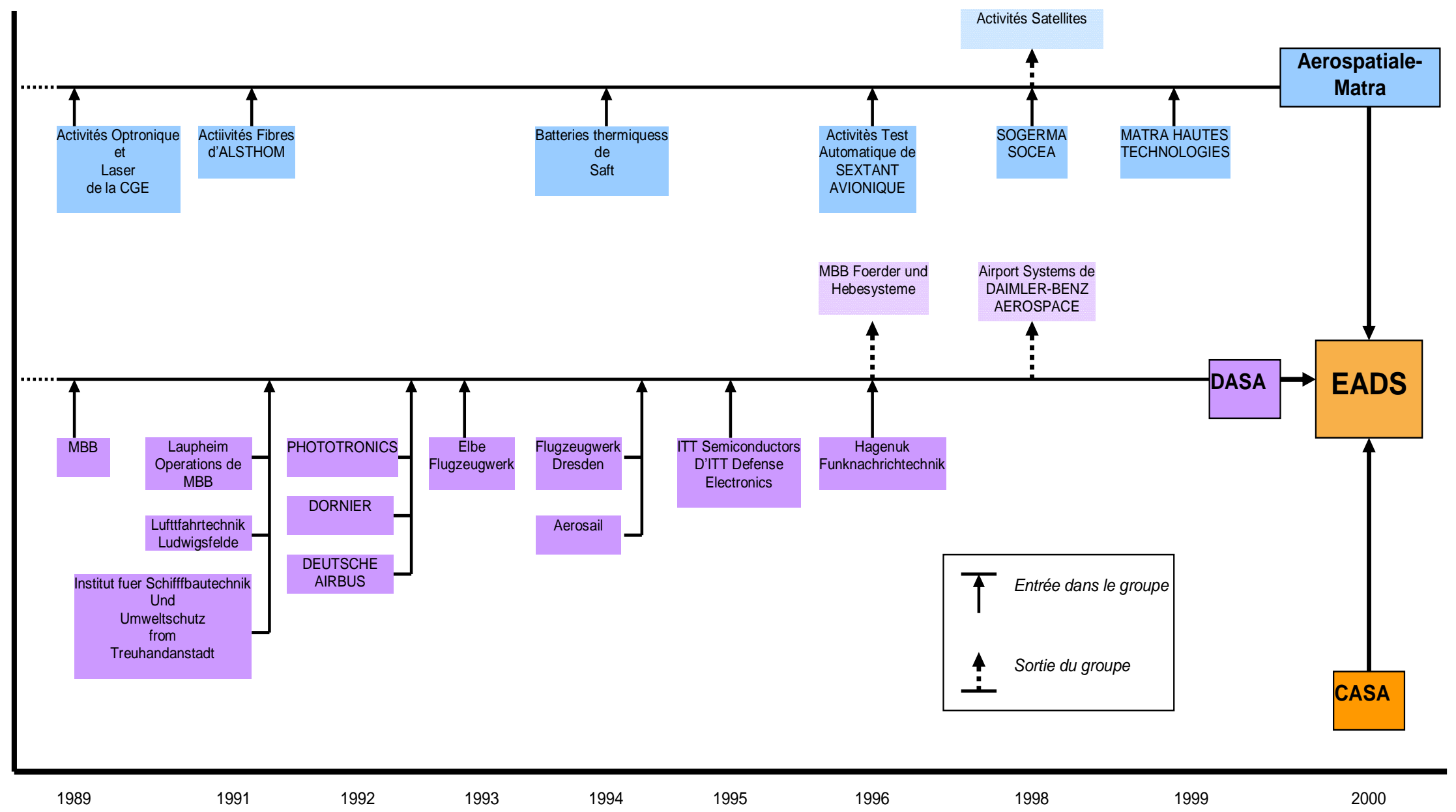

Source : Frigant, Kechidi, Talbot $(2006)^{16}$.

\section{Figure 2. La formation d'EADS}

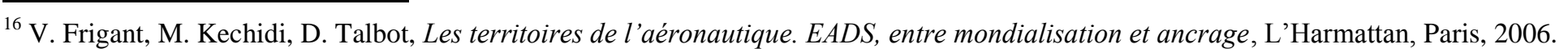


Avant de relever les principales caractéristiques du modèle d'organisation industriel d'Airbus, nous revenons sur l'évolution technique des principaux programmes. En effet, trajectoire d'innovation et trajectoire organisationnelle sont liées, illustrant une certaine histoire industrielle de l'avionneur européen fondée sur l'innovation et les ruptures de paradigme (Kechidi, 2008 ${ }^{17}$ ).

\section{2 - Les processus d'innovation : «tout à l'avant », « tout électrique », « tout composites »}

Les innovations technologiques ont en effet marqué l'évolution des programmes Airbus. Ces innovations constituent à chaque fois une rupture de paradigme au sens où elles introduisent de nouvelles architectures de produit. Ces choix technologiques ont induit des modifications profondes dans l'organisation industrielle de l'avionneur, mais également dans ses relations avec les entreprises sous-traitantes (Kechidi, 2008) ${ }^{18}$.

La complexification croissante des avions, résultat et moteur des processus d'innovation technique, est une donnée constante dans l'évolution des programmes Airbus. Si cette complexification touche aujourd'hui principalement l'avionique et les systèmes embarqués, elle concerne toute la conception et la production de l'avion depuis les premiers programmes. L'A300, premier biréacteur à large fuselage et long courrier, inaugure le poste de pilotage «tout à l'avant». Cette innovation permet de passer au pilotage à deux, nouveauté pour un gros porteur. Elle s'inscrit dans l'ère de la logique de marché : « un bon avion est un avion qui se vend » et toute innovation doit répondre à un besoin exprimé par les clients. Cette stratégie remplace l'avion des ingénieurs, le Concorde, un bel objet technique mais qui ne se vend pas. En termes d'externalisation, nous sommes dans une phase où Airbus réalise l'essentiel de l'avion en interne. L'externalisation porte pour l'essentiel sur du travail à façon. Les entreprises sous-traitantes produisent des composants à partir des études et des plans détaillés communiqués par l'avionneur. Le modèle industriel est celui d'une sous-traitance classique où les entreprises liées fonctionnent comme des ateliers externalisés qui fournissent des pièces et des composants au constructeur.

En 1984, l'introduction des commandes électriques de vol sur l'A320 ainsi qu'une nouvelle conception du cockpit constituent une véritable révolution technique. Elle permet de gagner en masse et en nombre d'équipements. Les commandes électriques et le pilotage automatique

\footnotetext{
${ }^{17}$ M. Kechidi, "Modularité, firme pivot et innovations : un nouveau modèle d'organisation industrielle pour Airbus?", Revue Français de Gestion Industrielle, vol. 27, n², 2008, p. $29-45$.

${ }^{18}$ Ibid.
} 
inaugurent l'arrivée massive de l'électronique et des systèmes embarqués. Ceci n'est pas sans conséquence sur l'organisation industrielle. C'est l'architecture d'ensemble de l'avion qui est repensée. La maîtrise des systèmes électriques devient un actif spécifique fondamental. Selon les programmes, ce ne sont pas toujours les mêmes modules électriques qui sont externalisés. Il y a là un souci net de conserver en interne des compétences majeures et de freiner les risques de dépendance vis-à-vis de fournisseurs externes.

Les familles A318/A319/A320/A321 et A330/A340 introduisent à la fin des années 1980 une quasi standardisation des équipements de cockpit. Les avions de la même famille possèdent le même tableau de bord, les mêmes procédures de pilotage, la même avionique et quasiment les mêmes systèmes. Ces configurations similaires permettent dès lors aux mêmes équipages de piloter tous les avions d'une même famille. Cet argument commercial procède également d'une rationalisation des processus de fabrication. Ainsi, en matière d'outillage, l'A321 réutilise la quasi-totalité des équipements développés pour l'A320.

Dans le cas de l'A380, les avancées technologiques sont des réponses aux problèmes posés par la taille et le poids de l'avion. Ces nouvelles contraintes ont nécessité le développement de sous-systèmes hydrauliques et électriques de transport de l'énergie qui ne génèrent pas des masses élevées ni des pertes de charges importantes. L'A380 accélère l'ère de «l'avion tout électrique ». Ce concept repose sur la suppression de la génération hydraulique centralisée, développée par Messier-Bugatti et Sofrance (Safran). Il a permis de résoudre les contraintes de charge en remplaçant des systèmes centralisés entièrement hydrauliques reliés par des systèmes électro-hydrauliques dédiés à chaque équipement. Cette innovation permet des gains de masse importants et une diminution des coûts de production et de maintenance.

L'autre innovation introduite sur l'A380 est l'électronique modulaire intégrée. Jusqu'au programme A340, les systèmes avioniques étaient composés d'un ensemble d'équipements numériques (des calculateurs) reliés et dédiés, chacun, à une seule fonction. L'ajout d'une fonction nouvelle à bord de l'avion nécessitait l'implantation de nouveaux calculateurs et de nouvelles liaisons. En d'autres termes, cette avionique, dite classique, est constituée d'un ensemble de ressources non partagées. Parce qu'elle induisait la multiplication des équipements, elle générait des charges et des coûts élevés. L'Avionique Modulaire Intégrée (AMI) adoptée par l'A380 (et par les B777 et 787) consiste à abandonner ce principe de ressource dédiée pour faire partager une même architecture matérielle à des applications différentes. Concrètement, un maximum de calculateurs de bord sont regroupés dans des modules électroniques communs à plusieurs fonctions. Le calculateur central permet d'héberger les fonctions de calcul de plus de 80 sous-systèmes de l'avion. Cette organisation 
informatique favorise une grande évolutivité des fonctionnalités logicielles dans la mesure où elle permet de modifier des éléments sans avoir à toucher à l'architecture informatique de l'avion.

Typiquement, on est en présence de technologies qui améliorent les performances de l'appareil tout en réduisant les coûts de fabrication et de maintenance ainsi que le poids des appareils. Sur le 787, le Common Core System permet de réduire ainsi le poids de l'avionique de près d'une tonne par rapport à un 767. Au plan industriel, le passage d'une logique «un calculateur pour chaque fonction » à une logique «un calculateur pour plusieurs fonctions » réduit le nombre d'intervenants dans la fabrication des modules électroniques et permet de déléguer la fonction de coordination à un acteur unique, une firme-pivot, qui va organiser la cascade de sous-traitance.

Enfin, même si l'A350 XWB adopte des technologies déjà éprouvées sur les précédents programmes, notamment l'A380 ou l'A400M, il pousse loin le concept tout électrique et tout composite. Par exemple, la part des matériaux composites est ainsi passée à $52 \%$ (contre 38\% sur la version avortée de l'A350) alors que les aluminiums et les lithiums restent à $20 \%$.

Cette rapide histoire technique des programmes successifs d'Airbus renseigne sur la trajectoire d'innovation qui caractérise les différents programmes d'Airbus. Cette trajectoire est également liée à des mutations dans le modèle d'organisation industrielle, notamment dans l'architecture des relations entre le constructeur et les différentes firmes qui participent au processus industriel.

\section{3- Le modèle d'organisation industrielle}

L'organisation industrielle d'Airbus repose sur l'instauration d'une division des tâches internes relativement stable aux niveaux national et international et fondée sur les compétences principales de chaque site de production. En revanche, la répartition des tâches entre Airbus et ses sous-traitants a profondément évoluée sous l'action conjuguée, d'une part d'un mouvement de recentrage et d'externalisation opéré par les constructeurs qui voit la fin de l'avionneur intégré et, d'autre part, d'un approfondissement de l'organisation modulaire qui provoque l'apparition de firmes-pivots dans le rang 1 de la supply chain.

\subsection{Une répartition entre les sites d'Airbus par compétences}

Le cycle de production d'un Airbus s'inscrit dans le cadre d'une quadruple division du travail :

- une division européenne du travail entre les quatre "NatCos" (Compagnies nationales) participant à EADS : Allemagne, Angleterre, Espagne, France ; 
- une répartition nationale de la charge revenant à firme nationale. Dans le cas d'Airbus France la production est ainsi répartie entre quatre sites de production (Toulouse, Nantes, Méaulte, Saint-Nazaire) ;

- une répartition des tâches entre les différentes usines au sein d'un même site. On compte par exemple quatre usines sur le site toulousain (Blagnac, Colomiers, SaintMartin du Touch et Saint-Eloi) ;

- une division du travail entre Airbus et un vaste réseau de sous-traitance constitué de firmes implantées en France ou à l'étranger.

Concernant la répartition européenne des charges, l'organisation la plus courante consiste à fabriquer les ailes en Grande Bretagne, l'empennage arrière en Espagne, le fuselage en Allemagne, l'avant de l'appareil ainsi que la section centrale du fuselage en France, l'assemblage final se faisant à Toulouse ou à Hambourg. La spécialisation par site européen est la suivante :

Tableau 1. Spécialisation des principaux sites de production d'Airbus SAS

\begin{tabular}{|c|c|}
\hline \multicolumn{2}{|c|}{ Airbus Deutschland } \\
\hline Bremen & éléments fuselage arrière \\
\hline Dresde & $\begin{array}{l}\text { production et assemblage des planchers des } \\
\text { avions }\end{array}$ \\
\hline Hambourg & $\begin{array}{l}\text { systèmes de pressurisation, conditionnement } \\
\text { d'air, assemblage fuselage arrière, } \\
\text { assemblage final des A318, A319, A321 }\end{array}$ \\
\hline Laupheim et Buxtehude & customisation \\
\hline Nordenham & usinage grandes pièces mécaniques \\
\hline Stade & $\begin{array}{l}\text { production des dérives et empennages } \\
\text { verticaux }\end{array}$ \\
\hline Varel & $\begin{array}{l}\text { traitement des pièces hautes précisions pour } \\
\text { fuselage }\end{array}$ \\
\hline \multicolumn{2}{|c|}{ Airbus Espana } \\
\hline Getafe & $\begin{array}{l}\text { production de panneaux en matériaux } \\
\text { composites, conception et assemblage final } \\
\text { des empennages horizontaux }\end{array}$ \\
\hline
\end{tabular}




\begin{tabular}{|c|c|}
\hline Illescas & $\begin{array}{l}\text { production de panneaux bruts en fibre de } \\
\text { carbone pour empennages horizontaux }\end{array}$ \\
\hline Puerto Real & $\begin{array}{l}\text { montage et usinage des composants en fibre } \\
\text { de carbone ou en matériaux aluminés pour } \\
\text { les empennages horizontaux }\end{array}$ \\
\hline \multicolumn{2}{|c|}{ Airbus France } \\
\hline Méaulte & usinage de pièces mécaniques pointe avant \\
\hline Nantes & $\begin{array}{l}\text { assemblage de tronçons mécanique et } \\
\text { composite, caisson central }\end{array}$ \\
\hline Saint-Nazaire & assemblage de tronçons fuselage central \\
\hline Toulouse & $\begin{array}{l}\text { conception avionique, conception et } \\
\text { production des mâts réacteurs, assemblage } \\
\text { A300/310, A320, A330/340, A380, A350, } \\
\text { essais en vol }\end{array}$ \\
\hline \multicolumn{2}{|c|}{ Airbus UK } \\
\hline Bristol - Filton & $\begin{array}{l}\text { conception des ailes, équipements en } \\
\text { systèmes électriques des ailes de l'A320, } \\
\text { partie de fuselage de l'A321, pièces pour les } \\
\text { ailes des A330/340 }\end{array}$ \\
\hline Chester - Broughton & production et assemblage des ailes \\
\hline
\end{tabular}

Source : site web Airbus SAS.

Selon le type d'avion, la répartition des tâches peut varier. Ainsi, les voilures produites à Chester des A300, A310, A330 et A340 sont d'abord acheminées à Brême pour recevoir des équipements avant d'être réexpédiées à Toulouse pour être montées, tandis que celles de l'A380 ne transiteront pas par l'Allemagne. Les A320 sont assemblés à Toulouse, tandis que les autres appareils de la famille (A318, A319, A321) le sont à Hambourg. Il faut voir dans cette duplication des sites d'assemblage la résurgence régulière des confrontations entre industriels européens, le partenaire allemand souhaitant obtenir la tâche très symbolique de l'assemblage final d'une partie des Airbus pour des raisons de prestige national, mais aussi parce que les compétences techniques et organisationnelles sont bien maîtrisées par les équipes hambourgeoises. 


\subsection{Stratégies de recentrage et d'externalisation chez les avionneurs : la fin de la firme} intégrée

Par le passé, les constructeurs aéronautiques concevaient, réalisaient et fabriquaient leurs appareils dans une très large mesure en interne. A partir des années 1980, on assiste à la fin du modèle de la firme intégrée. Concrètement, la part des achats dans le chiffre d'affaires d'Aérospatiale est passée de 40 à $70 \%$ entre 1980 et 1990. Toutefois, cette externalisation des activités secondaires a entraîné une multiplication du nombre de sous-traitants, un coût de suivi élevé et des difficultés pour s'assurer de la qualité des productions. Ces conséquences négatives d'une gestion décentralisée, mais également la perspective du développement de nouveaux programmes, ont alors contraint Airbus à faire évoluer sa stratégie au début des années 1990.

L'objectif pour Airbus est alors double. Il s'agit d'abord de réduire le nombre des soustraitants, l'ambition étant de passer de près de 700 sous-traitants à environ 120 sous-traitants de premier niveau. Ensuite, dans une optique de recherche d'aide au financement du développement en raison du désengagement de l'Etat, il s'agit de sélectionner des soustraitants dont la taille garantit une autonomie de gestion et des capacités financières autorisant un partage des risques. Se constitue progressivement autour de l'entreprise un réseau stable de partenaires aux activités complémentaires, dans lequel les relations durables sont contractualisées et des liens de partenariat instaurés avec des objectifs d'amélioration des performances et un partage des risques ${ }^{19}$.

Aujourd'hui, les avionneurs confient le design et la conception de parties entières de l'avion à des sous-traitants. Ils sont passés ainsi du statut de «constructeur aéronautique » à celui « d'assembleur de systèmes aéronautiques » (Cagli et al., 2012 ${ }^{20}$; Kechidi, 2013 ${ }^{21}$ ). Ainsi, Boeing et Airbus se positionnent à l'amont et à l'aval de la chaîne de valeur. Boeing soustraite plus de $70 \%$ (en valeur) des pièces de son Dreamliner. C'est la même approche pour l'A350 qui voit $50 \%$ des tâches de l'aérostructure confiées à des entreprises externes, parfois implantées dans des pays à bas coûts ou en zone dollars.

\footnotetext{
${ }^{19}$ La restructuration passe également par le désengagement de certains sites. Trois usines sont considérées comme «non prioritaires »: Saint-Nazaire Ville (850 emplois), qui verra son activité regroupée avec celle voisine de Gron, ainsi que Varel et Laupheim en Allemagne. Airbus souhaite voir des entreprises, notamment d'aérostructure à l'image de Latécoère ou de Spirit, prendre une part importante dans les investissements nouveaux que nécessite la reconversion de ces sites sur des activités composites.

${ }^{20}$ A. Cagli, M. Kechidi et R. Lévy, "Complex product and supplier interfaces in aeronautics", Journal of Manufacturing Technology Management, vol. 23, nº, 2012, p. 717-732.

${ }^{21}$ M. Kechidi, "From "aircraft manufacturer" to "architect-integrator": Airbus's industrial organization model", International Journal of Technology and Globalisation, Special Issue on: "The International Civil Aircraft Industry: Turbulence Ahead", vol. 7, ${ }^{\circ} 1 / 2,2013$, p. 8-22
} 
Clairement, le «Nouvel Airbus » annoncé par Power 8 est fondé sur un recentrage des activités sur le coeur de métier de l'entreprise. Ces activités renvoient à l'architecture globale cabine et avion, l'intégration des systèmes, l'assemblage, la customisation ainsi que les essais des équipements et autres composants jugés déterminants pour l'intégrité et la sécurité des appareils (comme par exemple certains systèmes électroniques, les commandes de vol ou les mâts réacteurs). Finalement, la segmentation stratégique des achats qui émerge comme mode d'organisation de l'outsourcing repose sur quatre niveaux :

- l'aérostructure ;

- les équipements ;

- les systèmes ;

- les composants et équipements aéronautiques.

\subsection{Approfondissement de l'organisation modulaire dans la supply chain : l'émergence des} firmes-pivots

La modularisation n'est pas un phénomène récent dans l'activité aéronautique, l'avion étant un produit modulaire par excellence. D'emblée elle a été au coeur des relations verticales entre donneurs et receveurs d'ordres (Larré, 1994) ${ }^{22}$. Les mutations récentes annoncent un approfondissement de cette organisation ainsi qu'une forte redistribution des rôles entre acteurs participant au processus de production de l'avion (Frigant, Talbot, 2005 ${ }^{23}$, Cagli et alii $\left.2009^{24}\right)$.

Dans un contexte de modularité pure, la démarche d'une firme architecte consiste à décomposer le produit final en une série de sous-ensembles indépendants dans leur conception et leur production et simultanément interdépendants lorsqu'il s'agit de les associer pour former le produit final. La modularisation se fonde alors sur une décomposition technique et cognitive des processus productifs (Ulrich, 1995) ${ }^{25}$. L'organisation des firmes doit être repensée (Langlois, $2003^{26}$; Frigant, 2005 ${ }^{27}$ ) : c'est l'avènement d'une organisation

\footnotetext{
${ }^{22} \mathrm{~F}$. Larré, Mécanismes et formes de coordination interentreprises, l'analyse d'un réseau de sous-traitance, thèse de doctorat en Sciences Economiques, Université des Sciences Sociales de Toulouse, mars 1994.

${ }^{23}$ V. Frigant, D. Talbot, "Technological determinism and modularity: lessons from a comparison between Aircraft and Auto Industries in Europe", Industry and Innovation, vol. 12, n³, 2005, pp. 337-335.

${ }^{24}$ Cagli A. Kechidi M. Lévy R." Gestion stratégique de la supply chain et firme-pivot dans le secteur aéronautique “, Revue Française de Gestion Industrielle, n 2, juin 2009 pp 89-106.

${ }^{25}$ K. Ulrich, "The role of product architecture in the manufacturing firm", Research Policy, vol. 24, n³, 1995, p. 419-40.

${ }^{26}$ R.N. Langlois, "The vanishing hand: the changing dynamics of industrial capitalism", Industrial and Corporate Change, vol. 12, $\mathrm{n}^{\circ} 2,2003$, p. 351-385.

27 V. Frigant, "Vanishing hand versus Systems integrators. Une revue de la littérature sur l'impact organisationnel de la modularitéc, Revue d'économie industrielle, vol. 109, n¹, 2005, p. 29-52.
} 
dans laquelle une firme architecte coordonne un ensemble de fournisseurs spécialisés sur des blocs de compétences particuliers (Cagli et alii., 2009) ${ }^{28}$.

Nous l'avons souligné, dans une organisation modulaire l'architecte confie à des firmes spécialisées la conception et la production de modules ou de composants du produit final $\left(\right.$ Sanchez, Mahoney, 1996) ${ }^{29}$. Son rôle de supervision consiste à contrôler la réalisation des sous-ensembles et à s'assurer de leur compatibilité via les interfaces avec les autres modules. Selon le degré de division technique du travail, l'architecte aura à contrôler un nombre plus ou moins important d'interfaces. Il s'assure que les caractéristiques des ensembles produits correspondent bien aux interfaces spécifiées ex ante.

L'approfondissement de l'organisation modulaire chez Airbus consiste principalement à confier à des entreprises spécialisées, les firmes-pivots (Cagli et al., 2009 ${ }^{30}$; Gilly et al., $2011^{31}$ ) ou "hub firm" (Jarillo, 1988) ${ }^{32}$ des ensembles de plus en plus importants. Plus la taille des ensembles et sous-ensembles confiés s'accroît et moins grande est la charge de coordination de l'architecte, le nombre d'interfaces à contrôler se réduisant avec le nombre de modules à recomposer. L'avantage économique de la modularisation est net en termes de réduction des coûts, grâce à la réduction du nombre de partenaires directs. La firme-pivot peut jouer à son tour un rôle de firme architecte pour les ensembles qui lui sont confiés. Son rôle de pivot réside dans le fait qu'elle va articuler les compétences techniques et organisationnelles d'autres participants au projet productif. Elle développe alors des compétences combinatoires. Elle détient des ressources (techniques, organisationnelles et financières) pour participer à la prise en charge en conception et en production d'un sousensemble technique majeur du produit final.

Sur cette base, la configuration «optimale » d'un réseau de sous-traitance serait un premier rang constitué de firmes-pivot pour un ensemble technico-industriel homogène (aérostructures, câblage électrique, systèmes embarqués, etc.), puis de sous-traitants de spécialité et de capacité. La caractéristique des firmes-pivot seraient de pouvoir partager les risques selon la formule, «l'équipement n'est payé que si l'avion est vendu ».

\footnotetext{
${ }^{28}$ A. Cagli, M. Kechidi, R. Lévy, "Gestion stratégique de la supply chain et firme-pivot dans le secteur aéronautique“, Revue Française de Gestion Industrielle, n², juin 2009, p. 89-106.

${ }^{29}$ R. Sanchez, J.T. Mahoney, "Modularity, flexibility, and knowledge management in product and organization design“, Strategic Management Journal, vol. 147, 1996, p.63-76.

${ }^{30}$ Op. cit.

${ }^{31}$ J.P. Gilly, D. Talbot, J.M. Zuliani, "Hub firms and the dynamics of regional innovation: case studies of Thales and Liebherr in Toulouse“, European Planning Studies, vol. 19, n¹2, p. 2009-2024.

32 J.C. Jarillo, “On strategic Networks”, Strategic Management Journal, vol. 9, 1988, p. 31-41.
} 
Figure 3. Les relations verticales au sein du secteur aéronautique
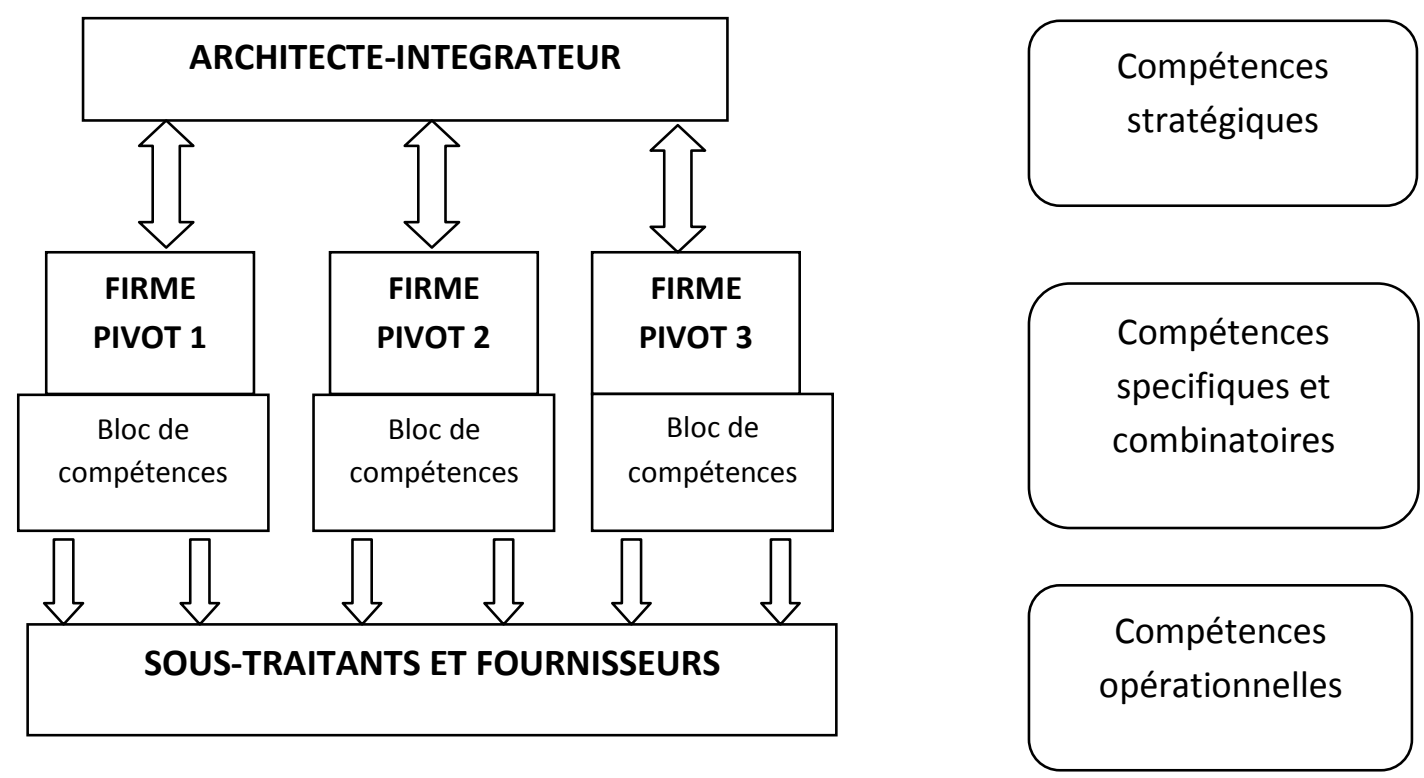

Source : Kechidi $\left(2013^{33}\right)$.

A titre d'exemple, Cagli et alii. (2009) montrent que la firme-pivot Goodrich se positionne sur de larges segments de la supply chain aéronautique. Elle intervient dans la production et la conception du moteur, des roues, des freins et du train d'atterrissage de l'avion. Elle participe également à la co-spécification des systèmes embarqués (notamment les systèmes de navigation, de communication et de maintenance). Ainsi, à travers ses différentes filiales, le groupe Goodrich collabore principalement avec Boeing (77 relations d'affaires contre 49 avec Airbus). Cela peut s'expliquer à la fois par la proximité géographique (collaboration entre deux groupes américains) et par le fait que Boeing externalise des segments et des volumes de production plus importants qu'Airbus.

\section{Conclusion}

Nous avons débuté ce travail en montrant que, à l'occasion du passage d'une logique d'arsenal à une logique de marché, on avait assisté à un désengagement des Etats. Toutefois, il faut rappeler que ce désengagement n'est que partiel, les politiques publiques marquant encore profondément l'évolution de cette industrie. C'est son caractère dual, entre civil et militaire, qui explique ce fait permanent, observable dans tous les pays. Aujourd'hui, l'intervention des Etats prend des formes multiples et indirectes. Leur poids reste considérable

\footnotetext{
${ }^{33}$ Op. cit.
} 
en tant qu'actionnaires et/ou clients et/ou financeurs. A titre d'exemple, lors de la dernière restructuration de l'actionnariat d'EADS opérée en avril 2013, la part des Etats passe de 19\% à $28 \%$ alors que les grands groupes privés tels que Lagardère et Daimler se retirent. Ainsi, «les Etats auront toujours quelque chose à dire parce que nous sommes des industries stratégiques pour leur sécurité », selon le directeur de la stratégie du groupe ${ }^{34}$.

Les mutations du modèle d'organisation industriel sont tout aussi importantes. Dans les développements précédents, nous avons soutenus l'idée qu'au fur et à mesure des différents programmes l'avionneur européen est passé d'un statut de constructeur aéronautique à celui d'architecte-intégrateur de systèmes. Cette évolution a été portée à la fois par le développement de la décomposition modulaire du processus de construction des avions, par une stratégie de recentrage sur le cœur de métier des architectes-intégrateurs, par l'externalisation de «packs techniques » entiers conduisant à l'émergence de firmes-pivots, ainsi que par des innovations radicales telles que les commandes électriques, les matériaux composites ou encore l'avionique modulaire intégrée.

Au-delà de ces phénomènes, il faut encore évoquer l'intensification de la concurrence, la financiarisation des comportements et l'internationalisation croissante des stratégies des entreprises comme autant d'éléments qui rejaillissent sur la configuration de cette industrie (Kechidi, Talbot, 2010) ${ }^{35}$. Concernant ce dernier point, les firmes aéronautiques françaises et européennes se sont internationalisées essentiellement du fait de la réalisation d'une part importante de leurs chiffres d'affaires hors de France ou d'Europe. Des travaux statistiques $\left(\right.$ Carrincazeaux, Frigant, 2007) ${ }^{36}$ ont montré que 1'internationalisation des firmes françaises s'opérait également à travers des implantations dans les principales régions productrices, sans que cela ne se traduise par une substitution des bases productives actuelles. Fait nouveau, l'internationalisation prend de plus en plus la forme d'une délocalisation de capacités de production européenne dans des pays tiers ou de localisation de nouvelles capacités dans ces pays. Pour le directeur de la stratégie de EADS «nous devons nous rapprocher de nos marchés et mieux équilibrer la répartition mondiale de nos sites au regard de nos ventes (...). Il ne s'agit pas de transférer nos usines, mais d'associer les pays émergents à notre croissance

\footnotetext{
${ }^{34}$ Cité par l'AFP dans une dépêche du 6 décembre 2012.

${ }^{35}$ M. Kechidi, D. Talbot, "Institutions and coordination: what is the contribution of a proximity-based analysis? The case of Airbus and its relations with the subcontracting network", International Journal of Technology Management, vol. 50, n³/4, 2010, p. 285-299.

${ }^{36}$ C. Carrincazeaux, V. Frigant, "The internationalization of French aerospace Industry: to what extent were the 1990s a break with the Past”, Competition and Change, vol. 11, n³, 2007, p. 260-284.
} 
et de positionner nos sites européens sur les activités à forte valeur ajoutée ${ }^{37}$. On peut distinguer plusieurs explications à cette (re) localisation. Il y a d'abord des motifs liés aux coûts. L'importance donnée à la maîtrise des coûts conduit les industriels à délocaliser des activités de production d'ensembles ou de composants issus de technologies banalisées (essentiellement en aérostructures et en câblage électrique). Ces productions sont localisées dans des pays qui proposent un rapport favorable coût/qualité de la main d'œuvre. Plusieurs firmes françaises (Latécoère, Aérolia, Indraero, Creuzet, etc.) sont engagées dans une délocalisation progressive d'une partie de leur production essentiellement au Maghreb, en Tchéquie et en Roumanie. Ces délocalisations sont qualifiées de near shore. Les délocalisations off shore concernent, pour les mêmes raisons, les implantations au Mexique, au Vietnam ou à Singapour. Ces localisations permettent également de «dollariser » les coûts d'approvisionnement, notamment dans un contexte de parité défavorable euro/dollar. Viennent aussi les motifs liés au développement de certains marchés nationaux. Ainsi s'explique l'implantation de la chaine d'assemblage d'A320 à Tianjin (Chine) et à Mobile (Etats-Unis).

Ces localisations industrielles inaugurent une nouvelle configuration de l'industrie au plan mondial, configuration qui va à l'avenir encore évoluer du fait de l'arrivée de nouveaux acteurs. La menace de nouveaux entrants sur le segment 90-140 passagers est aujourd'hui manifeste. La Chine, le Brésil, le Japon, le Canada ainsi que la Russie semblent développer des stratégies qui, à terme, menacent le duopole Airbus-Boeing. A très moyen terme, l'hypothèse d'un troisième, voire d'un quatrième acteur, est devenue largement réaliste.

\footnotetext{
${ }^{37}$ La lettre institutionnelle de EADS, $n^{\circ} 4$ juin 2011.
} 